\title{
Time to Treatment Discontinuation in German Patients with Schizophrenia: Long-Acting Injectables versus Oral Antipsychotics
}

\author{
Jörg Mahlich ${ }^{1,2} \mathbb{E} \cdot$ Kerstin Olbrich ${ }^{1} \cdot$ Adrian Wilk $^{3} \cdot$ Antonie Wimmer $^{4} \cdot$ Claus Wolff-Menzler $^{5}$
}

Accepted: 18 November 2020 / Published online: 17 December 2020

(c) The Author(s) 2020

\begin{abstract}
Background and Objective Long-acting injectable antipsychotics (LAIs) are associated with better treatment adherence and persistence than oral antipsychotics (OAPs) in patients with schizophrenia. However, real-world evidence assessing the impact of treatment with LAIs in Germany is limited. To fill this gap, we compared antipsychotic medication adherence and risk of treatment discontinuation (TD) among schizophrenia patients newly initiated on LAI or who switched their OAP regimen (overall cohort; OC).

Methods Claims data of German schizophrenia patients who initiated LAIs or switched their OAP during 2012-2016 (index date) were retrospectively analyzed. Treatment switch was defined as add-on medication to existing prescription or terminating the existing prescription and initiating another OAP. Adherence and time to treatment discontinuation (TTD) were estimated. Determinants of treatment discontinuation were analyzed using two Cox regression models. Model 1 controlled for age, sex, and Charlson Comorbidity Index (CCI); model 2 also included insurance status, and medication, visit, and psychiatric inpatient stay costs. Sensitivity analysis on patients who terminated existing prescriptions and initiated new OAPs (complete switch cohort; CSC) was performed.

Results In OC $(n=2650)$, LAI users had better adherence (35.4\% vs. $11.6 \%)$, persistence (no 60 -day gap; $40.7 \%$ vs. $19.8 \%$ ), and longer TTD (median [95\% confidence interval (CI)] 216 [193-249] vs. 50 [46-56] days) than OAP users. OAP usage (hazard ratio [HR] 1.89, 95\% CI 1.73-2.06; $p<0.001)$ and greater CCI (HR 1.04, 95\% CI 1.00-1.07; $p=0.023$ ) were associated with greater risk of TD in model 1. Model 2 showed similar results. LAI users in CSC also had better adherence, persistence, and longer TTD. In CSC too, OAP usage and greater CCI were associated with greater risk of TD in model 1, but only CCI was significant in model 2 . Higher pre-index psychiatric inpatient costs were associated with lower risk of TD (HR 0.99, 95\% CI 0.98-1.00; $p=0.014$ ).

Limitations Inherent limitations of claims data and lack of control on OAP administration may have influenced the results. Conclusion This real-world study associates LAIs with better medication adherence and lower antipsychotic discontinuation risk than OAPs.
\end{abstract}

\section{Introduction}

Schizophrenia is a severe mental disorder with characteristics that include disruptions in thinking, sense of self, perception, and language [1]. The Robert Koch Institute has reported the prevalence of schizophrenia in Germany to be

Supplementary Information The online version contains supplementary material available at https://doi.org/10.1007/s4026 1-020-00990-8.

Jörg Mahlich

joerg.mahlich@gmail.com

Extended author information available on the last page of the article between 0.80 and $1 \%$ [2]. The negative effect of schizophrenia on cognitive performance [3] impairs daily activities, work productivity, and social functioning [4], resulting in increased economic burden. The annual economic burden of schizophrenia for Germany was estimated to be €9.6-13.5 billion in 2008 [5].

Pharmacologic treatment remains the cornerstone of the management of schizophrenia [6]. However, the efficacy of antipsychotics depends on medication adherence [7], which is low among patients with schizophrenia [8, 9]. Evidence shows non-adherence to be associated with a greater risk of hospitalization in the USA [10-13] and Europe [14, 15]. A systematic review by Higashi et al. [16] also reported hospitalization to be a major consequence of non-adherence 


\section{Key Points}

Switch from an oral antipsychotic to a long-acting injectable antipsychotic was associated with better treatment persistence and lower risk of treatment discontinuation than a switch from an oral antipsychotic to another oral antipsychotic in a real-world cohort of German patients with schizophrenia.

Patients suffering from comorbidities also had a higher risk of treatment discontinuation.

among patients with schizophrenia. Thus, indirectly, treatment costs could be elevated by hospitalization as it is a major driver of healthcare costs, contributing to $43 \%$ of the total direct medical costs associated with the treatment of German patients with schizophrenia [5].

Usage of long-acting injectable antipsychotics (LAIs) is one of the strategies employed to improve adherence in patients with schizophrenia, as LAIs have been shown to increase medication adherence and reduce hospitalization [17-23], as well as to be more cost-effective compared with oral antipsychotics (OAPs) [23-28]. Recent studies have evaluated the difference in medication adherence between LAIs and OAPs using claims data from the USA [20, 25, 29]. Greene et al. [20] reported that LAI users had a higher mean proportion of days covered (PDC) than OAP users ( 0.55 vs. $0.50 ; p<0.001)$, while OAP users had a greater risk of treatment discontinuation (hazard ratio [HR] 1.20, 95\% confidence interval $[\mathrm{CI}] 1.12-1.28 ; p<0.001)$. Pilon et al. [25] reported the odds of being adherent to the prescribed treatment $(\mathrm{PDC} \geq 0.8$ ) were higher among users of secondgeneration antipsychotic (SGA) LAIs compared with OAP users (odds ratio [OR] 1.28, 95\% CI 1.18-1.40; $p<0.001$ ). Joshi et al. [29] reported similar results as users of paliperidone palmitate LAI were more adherent than OAP users (PDC $\geq 0.8: 48.1 \%$ vs. $32.6 \% ; p<0.001$ ).

Although evidence supporting the use of LAIs to improve medication adherence is available elsewhere, similar studies have not been conducted in Germany. To address this knowledge gap, we compared medication adherence and persistence of LAIs and OAPs using data from a large German claims database.

\section{Methods}

\subsection{Data Source}

De-identified patient data were obtained from a claims database provided by Team Gesundheit, Essen, Germany. This database comprises comprehensive patient-level electronic records of health insurance claim information on inpatient, outpatient, and prescription drugs. Over 3.7 million members of the German statutory health insurance are covered by this database. Moreover, the age and sex distribution resemble that of the general population insured with statutory health insurance. This database has been extensively utilized for health services research [30-32]. In 2018, around $87.7 \%$ of the population (72.8 million [33] among 83 million people [34]) was covered by statutory health insurance, while $12.3 \%$ of the population was privately insured.

\subsection{Study Population}

This retrospective cohort study included patients who were diagnosed with schizophrenia according to ICD-10-GM (International Classification of Diseases German Modification, 10th Revision) code F20.x (see Online Supplementary Material (OSM), Table 1) from 2011 to 2016 with a maximum follow-up till 2017 (OSM Fig. 1). Diagnoses made in the inpatient setting (primary and secondary diagnosis) or outpatient setting (verified diagnosis) were considered. Patients who were new LAI users, as defined by a documented LAI prescription, or had switched their OAP during 2012-2016, were identified. Treatment switch for OAP users was defined as add-on medication to the existing OAP prescription or termination of the existing OAP prescription and initiation of a new OAP. The following LAIs were identified: fluphenazine, haloperidol, flupenthixol, zuclopenthixol, fluspirilene, olanzapine, risperidone, aripiprazole, and paliperidone (OSM Table 2). The identified OAPs are reported in OSM Table 3. The index date was defined as the first prescription of the LAI for LAI users and as the first prescription after switching from one OAP regimen to another for OAP users.

An ICD-10 F20.x diagnosis in the quarter of the index date or in the preceding quarter, age $\geq 18$ years at the index date, and presence of at least 365 days of continuous enrollment prior to and after the index date was essential for inclusion in the study. Patients who were prescribed an LAI before 2012 and patients who were identified with certain comorbidities such as attention deficit/hyperactivity disorder (ICD-10 F90.x-F98.x), epilepsy (ICD-10 G09.x, G40.x, G41.x, I69.4, and O99.3), or dementia (ICD-10 F0.x, G30.x, G31.x, G10.x, G20.X, B22.0, and E75.6) in the quarter of the index date or in the preceding quarter were excluded (Fig. 1) as patients with these comorbidities often receive off-label antipsychotic medications $[35,36]$.

\subsection{Covariates}

The selection of covariates was conducted in line with previous studies [31, 37]. Age, sex, common comorbidities, 
and the Charlson Comorbidity Index (CCI) [38] were the baseline covariates of interest. The CCI included 19 comorbidities as reported in OSM Table 4 (myocardial infarction, congestive heart failure, peripheral vascular disease, cerebrovascular disease, dementia, chronic pulmonary disease, rheumatic disease, peptic ulcer disease, mild liver disease, diabetes without chronic complication, diabetes with chronic complication, hemiplegia or paraplegia, renal disease, tumors without metastasis, lymphoma, leukemia, moderate or severe liver disease, metastatic solid tumors, and AIDS/ HIV) and assigned a weight between 1 and 6 to each comorbidity. Higher CCI indicates greater patient morbidity.

\subsection{Outcomes}

Medication adherence was assessed using PDC in the 1 year after the index date. PDC was defined as the number of available days of index therapy divided by 365 (number of days in a year) [39]. The days' supply for each LAI claim was set to the minimum time between injections as per the individual drug's labeled dosing schedule [40]. For OAPs, PDC was calculated using the days' supply based on the daily defined doses as reported by the German Institute of Medical Documentation and Information [41]. Adherence was defined as PDC $\geq 0.80$. Persistence was defined in three ways: proportion of patients with no 30-day supply gap, no 60-day gap, and no 90-day gap during 1 year after the index date.

Time to treatment discontinuation was calculated with the Kaplan-Meier method. The persistence period was defined as the time from treatment initiation (index date) until discontinuation of the index medication. Patients were considered to have discontinued the index treatment if: (1) a gap exceeding 60 days (grace period) after the end of supply of the index medication was found, or (2) the patient switched from the index medication to another treatment(s). This definition of drug survival was consistent with that employed in other studies on persistence [37, 42].

\subsection{Statistical Analysis}

We analyzed all the outcomes in two cohorts: an overall cohort (OC) and a subset of the OC named the complete switch cohort (CSC). Primary analysis comprised patients in the OC, i.e. those who initiated a LAI or switched their OAP. Sensitivity analysis was performed using the CSC, i.e. those who initiated a LAI and those who terminated their existing OAP prescription and initiated a new OAP. Patients who had a medication added onto their existing OAP prescription were excluded from the sensitivity analysis.

Means and standard deviations for continuous variables and counts and percentages for categorical variables were calculated. The Kaplan-Meier method was used to estimate time to antipsychotic medication discontinuation. Cox regression analysis was performed to examine the association between time to antipsychotic medication discontinuation and usage of OAP or LAI. Cox regression was performed using two approaches. In the first approach, the model was adjusted for age, sex, and CCI, while in the second approach the model was adjusted for age, sex, CCI, insurance status, medication costs, visit costs, and psychiatric inpatient stay costs. All analyses were performed using SAS software (version 9.4; SAS Institute Inc., Cary, NC, USA).

\section{Results}

\subsection{Population Characteristics}

Among the 27,363 patients diagnosed with schizophrenia, 2650 were eligible for the study (Fig. 1). The OC included $1132(42.7 \%)$ LAI users and 1518 (57.3\%) OAP users. Table 1 shows the baseline characteristics. OAP users were older than LAI users (mean [SD] age 46.9 [14.2] vs. 42.3 [14.5] years). Males comprised a greater proportion of the patients in both groups. A greater proportion of OAP users had compulsory insurance than LAI users (28.5\% vs. $21.4 \%$ ). LAIs were prescribed more to young individuals while OAPs were prescribed more to the elderly. A greater proportion of OAP users were afflicted with comorbidities such as diabetes, liver disease, chronic pulmonary disease, and renal disease compared with LAI users, and this reflected in the higher CCI as well (mean [SD] 0.9 [1.4] vs. 0.6 [1.1]). However, use of substances such as alcohol, opioids, etc. were greater in LAI users than in OAP users.

The initial antipsychotic agent was prescribed more in the LAI group than in the OAP group during the post-index period (mean [SD] 5.6 [4.5] vs. 4.0 [3.3]). SGAs were widely prescribed in both the groups in both the pre- and post-index periods. Paliperidone (36.5\%) and risperidone (24.7\%) were the most prescribed LAIs during the postindex period (Table 2).

The CSC comprised 533 (69.9\%) LAI users and 230 (30.1\%) OAP users. Similar to the OC, OAP users in the CSC were older than LAI users (mean [SD] age 47.3 [15.6] vs. 44.1 [15.0] years). The majority of users in both groups were male. A greater proportion of OAP users had compulsory insurance than LAI users (31.7\% vs. $22.0 \%)$. CCI was higher among OAP users than LAI users (mean [SD] 1.0 [1.6] vs. 0.6 [1.2]) (Table 1). SGAs were widely prescribed in both the groups in both the pre- and post-index periods. Paliperidone (35.5\%) and risperidone (23.5\%) were the most prescribed LAIs during the post-index period (Table 2). 
Fig. 1 Data set for analysis. $A D H D$ attention-deficit/ hyperactivity disorder, $I C D$ International Classification of Diseases, $L A I$ long-acting injectable antipsychotic, $O A P$ oral antipsychotic

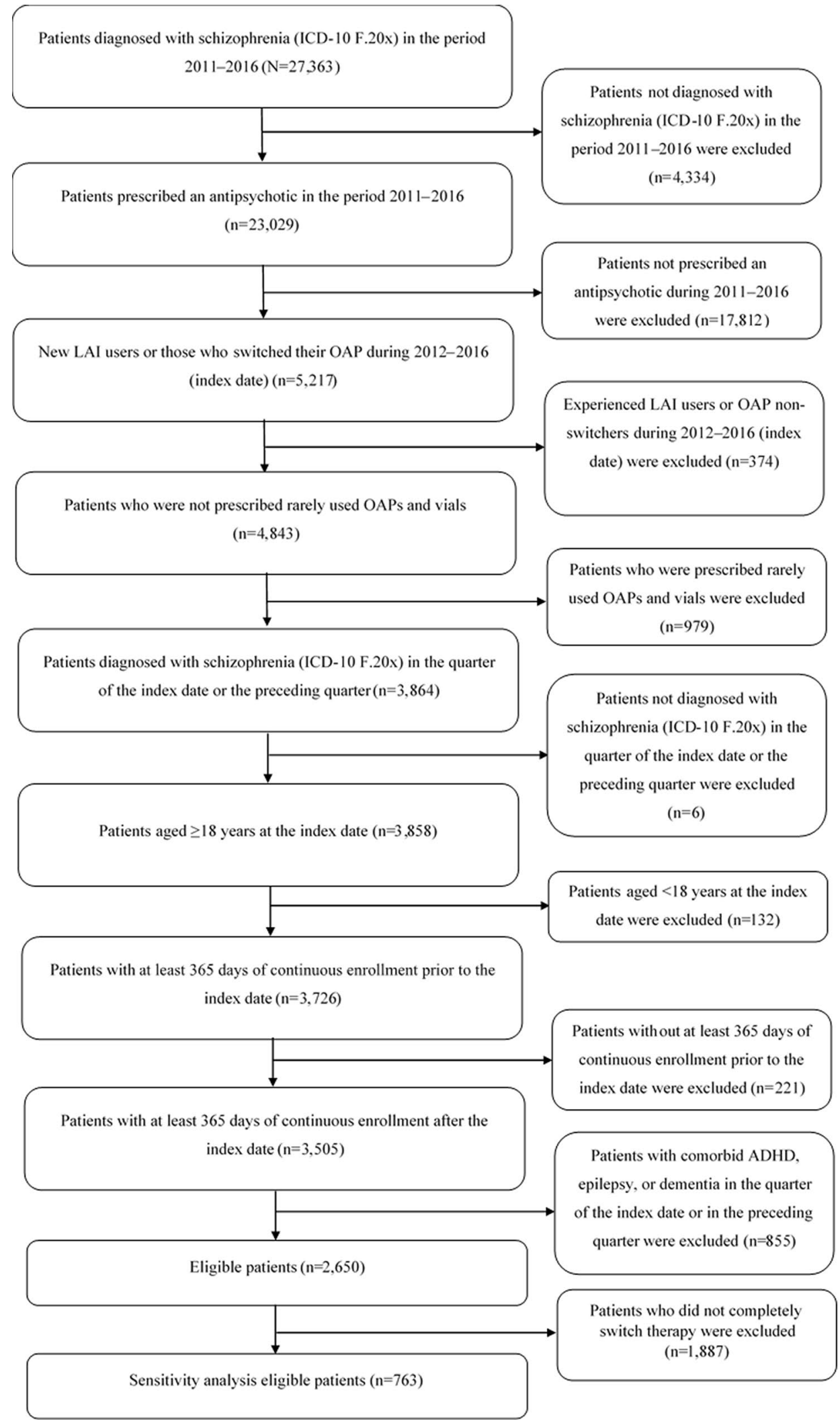


Table 1 Baseline characteristics

\begin{tabular}{|c|c|c|c|c|}
\hline \multirow[t]{2}{*}{ Parameter } & \multicolumn{2}{|l|}{ Overall cohort } & \multicolumn{2}{|c|}{ Complete switch cohort } \\
\hline & LAI $(n=1132)$ & Oral $(n=1518)$ & LAI $(n=533)$ & Oral $(n=230)$ \\
\hline Age, years, mean (SD) & $42.3(14.5)$ & $46.9(14.2)$ & $44.1(15.0)$ & $47.3(15.6)$ \\
\hline \multicolumn{5}{|l|}{ Age group, $\boldsymbol{n}(\%)$} \\
\hline $18-24$ & $102(9.0)$ & $49(3.2)$ & $40(7.5)$ & $14(6.1)$ \\
\hline $25-34$ & $324(28.6)$ & $293(19.3)$ & $139(26.1)$ & $40(17.4)$ \\
\hline $35-44$ & $227(20.1)$ & $331(21.8)$ & $97(18.2)$ & $42(18.3)$ \\
\hline $45-54$ & $232(20.5)$ & $412(27.1)$ & 117 (21.9) & $63(27.4)$ \\
\hline $55-64$ & $161(14.2)$ & $259(17.1)$ & $85(16.0)$ & $37(16.1)$ \\
\hline$\geq 65$ & $86(7.6)$ & $174(11.5)$ & $55(10.3)$ & $34(14.8)$ \\
\hline \multicolumn{5}{|l|}{ Sex, $n(\%)$} \\
\hline Male & $634(56.0)$ & $780(51.4)$ & $291(54.6)$ & $118(51.3)$ \\
\hline Female & $498(44.0)$ & 738 (48.6) & $242(45.4)$ & $112(48.7)$ \\
\hline \multicolumn{5}{|l|}{ Index year, $\boldsymbol{n}(\%)$} \\
\hline 2012 & $254(22.4)$ & $586(38.6)$ & $127(23.8)$ & $96(41.7)$ \\
\hline 2013 & $212(18.7)$ & $359(23.6)$ & $92(17.3)$ & $50(21.7)$ \\
\hline 2014 & $245(21.6)$ & $250(16.5)$ & $115(21.6)$ & $38(16.5)$ \\
\hline 2015 & $212(18.7)$ & $167(11.0)$ & $95(17.8)$ & $23(10.0)$ \\
\hline 2016 & $209(18.5)$ & $156(10.3)$ & $104(19.5)$ & $23(10.0)$ \\
\hline \multicolumn{5}{|l|}{ Insurance status, $n$ (\%) } \\
\hline No compulsory insurance & $4(0.4)$ & $9(0.6)$ & $1(0.2)$ & $1(0.4)$ \\
\hline Compulsory insurance & $242(21.4)$ & $432(28.5)$ & $117(22.0)$ & $73(31.7)$ \\
\hline Voluntary insurance & $104(9.2)$ & $136(9.0)$ & $40(7.5)$ & $18(7.8)$ \\
\hline Unemployed person & $192(17.0)$ & $110(7.2)$ & $96(18.0)$ & $24(10.4)$ \\
\hline Pension claimant & $8(0.7)$ & $4(0.3)$ & $3(0.6)$ & $1(0.4)$ \\
\hline Retired & $398(35.2)$ & $610(40.2)$ & $199(37.3)$ & $75(32.6)$ \\
\hline Special group of persons & $13(1.1)$ & $18(1.2)$ & $5(0.9)$ & $2(0.9)$ \\
\hline Family member & $106(9.4)$ & $132(8.7)$ & $48(9.0)$ & $24(10.4)$ \\
\hline Unknown & $65(5.7)$ & $67(4.4)$ & $24(4.5)$ & $12(5.2)$ \\
\hline CCI, mean (SD) & $0.6(1.1)$ & $0.9(1.4)$ & $0.6(1.2)$ & $1.0(1.6)$ \\
\hline \multicolumn{5}{|l|}{ Comorbidities, $n$ (\%) } \\
\hline MI & $7(0.6)$ & $14(0.9)$ & $4(0.8)$ & $8(3.5)$ \\
\hline $\mathrm{CHF}$ & $34(3.0)$ & $51(3.4)$ & $20(3.8)$ & $13(5.7)$ \\
\hline Peripheral vascular disease & $19(1.7)$ & $56(3.7)$ & $9(1.7)$ & $11(4.8)$ \\
\hline Cerebrovascular disease & $32(2.8)$ & $62(4.1)$ & $14(2.6)$ & $9(3.9)$ \\
\hline Dementia & $8(0.7)$ & $6(0.4)$ & $3(0.6)$ & $2(0.9)$ \\
\hline Chronic pulmonary disease & $184(16.3)$ & $295(19.4)$ & $83(15.6)$ & $47(20.4)$ \\
\hline Rheumatic disease & $15(1.3)$ & $20(1.3)$ & $6(1.1)$ & $2(0.9)$ \\
\hline Peptic ulcer disease & $12(1.1)$ & $18(1.2)$ & $7(1.3)$ & $5(2.2)$ \\
\hline Mild liver disease & $88(7.8)$ & $164(10.8)$ & $35(6.6)$ & $24(10.4)$ \\
\hline Diabetes without chronic complication & $105(9.3)$ & $223(14.7)$ & $50(9.4)$ & $44(19.1)$ \\
\hline Hemiplegia or paraplegia & $6(0.5)$ & $16(1.1)$ & $2(0.4)$ & $1(0.4)$ \\
\hline Renal disease & $28(2.5)$ & $65(4.3)$ & $13(2.4)$ & $11(4.8)$ \\
\hline Diabetes with chronic complication & $29(2.6)$ & $76(5.0)$ & $18(3.4)$ & $14(6.1)$ \\
\hline Tumor & $22(1.9)$ & $61(4.0)$ & $14(2.6)$ & $14(6.1)$ \\
\hline Leukemia & $1(0.1)$ & $4(0.3)$ & $1(0.2)$ & $0(0.0)$ \\
\hline Lymphoma & $3(0.3)$ & $3(0.2)$ & $2(0.4)$ & $0(0.0)$ \\
\hline Moderate or severe liver disease & $2(0.2)$ & $2(0.1)$ & $2(0.4)$ & $1(0.4)$ \\
\hline Metastatic solid tumor & $1(0.1)$ & $5(0.3)$ & $1(0.2)$ & $1(0.4)$ \\
\hline AIDS/HIV & $2(0.2)$ & $2(0.1)$ & $1(0.2)$ & $0(0.0)$ \\
\hline
\end{tabular}


Table 1 (continued)

\begin{tabular}{|c|c|c|c|c|}
\hline \multirow[t]{2}{*}{ Parameter } & \multicolumn{2}{|l|}{ Overall cohort } & \multicolumn{2}{|c|}{ Complete switch cohort } \\
\hline & LAI $(n=1132)$ & Oral $(n=1518)$ & LAI $(n=533)$ & Oral $(n=230)$ \\
\hline Use of alcohol & $206(18.2)$ & $165(10.9)$ & $92(17.3)$ & $30(13.0)$ \\
\hline Use of opioids & $32(2.8)$ & $25(1.6)$ & $15(2.8)$ & $8(3.5)$ \\
\hline Use of cannabinoids & $211(18.6)$ & $92(6.1)$ & $102(19.1)$ & $20(8.7)$ \\
\hline Use of sedatives or hypnotics & $56(4.9)$ & $58(3.8)$ & $18(3.4)$ & $7(3.0)$ \\
\hline Use of cocaine & $22(1.9)$ & $9(0.6)$ & $9(1.7)$ & $3(1.3)$ \\
\hline Use of other stimulants, including caffeine & $84(7.4)$ & $27(1.8)$ & $38(7.1)$ & $10(4.3)$ \\
\hline Use of hallucinogens & $7(0.6)$ & $5(0.3)$ & $5(0.9)$ & $0(0.0)$ \\
\hline Use of tobacco & $269(23.8)$ & $320(21.1)$ & $124(23.3)$ & $46(20.0)$ \\
\hline Use of volatile solvents & $1(0.1)$ & $1(0.1)$ & $0(0.0)$ & $0(0.0)$ \\
\hline $\begin{array}{l}\text { Multiple drug use and use of other psychoactive } \\
\text { substances }\end{array}$ & $188(16.6)$ & $108(7.1)$ & 78 (14.6) & $18(7.8)$ \\
\hline
\end{tabular}

$A D H D$ attention deficit/hyperactivity disorder, $A I D S$ acquired immunodeficiency syndrome, $C C I$ Charlson Comorbidity Index, $C H F$ congestive heart failure, $H I V$ human immunodeficiency virus, $L A I$ long-acting injectable antipsychotic, $M I$ myocardial infarction, $S D$ standard deviation

\subsection{Outcomes}

\subsubsection{Adherence and Persistence}

LAI users had better antipsychotic medication adherence than OAP users in the OC (35.4\% vs. $11.6 \%$ and mean [SD] PDC: 0.6 [0.3] vs. 0.3 [0.3]). In addition, a greater proportion of LAI users were observed to be persistent with their medication compared with OAP users (no 30-day gap: $26.4 \%$ vs. $9.6 \%$, no 60 -day gap: $40.7 \%$ vs. $19.8 \%$, and no 90-day gap: $48.2 \%$ vs. $28.3 \%$; Table 3 ).

Similar results were observed in the CSC. LAI users had better antipsychotic medication adherence than OAP users $(33.0 \%$ vs. $21.7 \%$ and mean [SD] PDC: 0.6 [0.3] vs. $0.4[0.3])$. Although the difference was not as great as that observed in the OC, a greater proportion of LAI users in the CSC were observed to be persistent with their medication compared with OAP users (no 30-day gap: $25.9 \%$ vs. $18.7 \%$, no 60 -day gap: $38.1 \%$ vs. $33.0 \%$, and no 90 -day gap: $46.0 \%$ vs. $42.6 \%$; Table 3 ).

\subsubsection{Time to End of Therapy}

A greater proportion of LAI users than OAP users were censored $(22.2 \%$ vs. $9.2 \%)$ in the OC. The median $[95 \% \mathrm{CI}]$ time to end of therapy was also longer in the LAI group than the OAP group (216 [193-249] vs. 50 [46-56]) days (Fig. 2). Presence of a $>60$-day gap in treatment was a major reason for end of therapy among LAI users, while OAP users were majorly considered to have discontinued therapy as they had used another agent after their last documented prescription (Table 4).

Cox regression models were used to identify the factors associated with a lower risk of discontinuation of antipsychotic treatment in the OC. The variables age, sex, and CCI are described in Table 1, while insurance status, medication costs, visit costs, and psychiatric inpatient stay costs are described in OSM Table 5. In model 1, age, sex, and CCI were controlled. Here, the factors associated with greater risk of antipsychotic discontinuation were usage of OAPs (hazard ratio [HR] 1.89; 95\% CI 1.73-2.06; $p<0.001$ ) and greater CCI (HR 1.04; 95\% CI 1.00-1.07; $p=0.023$ ). In model 2, age, sex, CCI, insurance status, medication costs, visit costs, and psychiatric inpatient stay costs were controlled. Here too, OAP usage (HR 1.87; 95\% CI 1.70-2.05; $p<0.001$ ) and greater CCI (HR 1.04; 95\% CI 1.00-1.08; $p=0.026)$ were associated with greater risk of antipsychotic discontinuation (Table 5).

In the CSC, the median [95\% CI] time to end of therapy was longer in the LAI group than the OAP group (211 [163-247] vs. 119.5 [86-157]) days (Fig. 3). Unlike in the OC, similar proportions of LAI and OAP users ended therapy due to presence of a $>60$-day gap in treatment and usage of another agent after their last documented prescription (Table 4). The factors associated with greater risk of antipsychotic discontinuation in model 1 were usage of OAPs (HR 1.19; 95\% CI 1.00-1.42; $p=0.05$ ) and greater CCI (HR 1.09; 95\% CI 1.02-1.16; $p=0.007)$. In model 2 , greater CCI (HR 1.08; 95\% CI 1.01-1.15; $p=0.032$ ) was associated with greater risk of antipsychotic discontinuation. OAPs only had a numerically bigger risk of treatment discontinuation over LAIs in model 2 (HR 1.08; 95\% CI $0.89-1.31$ ). Greater pre-index psychiatric inpatient stay costs were associated with a lower risk of antipsychotic discontinuation (HR 0.99; 95\% CI 0.98-1.00; $p=0.014$ ) (Table 5). 
Table 2 Medication characteristics and changes from pre-index to post-index period

\begin{tabular}{|c|c|c|c|c|}
\hline \multirow[t]{2}{*}{ Parameter } & \multicolumn{2}{|l|}{ Overall cohort } & \multicolumn{2}{|c|}{ Complete switch cohort } \\
\hline & LAI $(n=1,132)$ & Oral $(n=1,518)$ & LAI $(n=533)$ & Oral $(n=230)$ \\
\hline $\begin{array}{l}\text { Number of days between first and second prescription among patients } \\
\text { with second prescription, mean (SD) }\end{array}$ & $53.6(60.8)$ & $63.7(70.5)$ & $58.7(67.8)$ & $46.2(37.9)$ \\
\hline \multicolumn{5}{|l|}{ Number of injections of initial agent, mean (SD) } \\
\hline During post-index period & $12.8(10.6)$ & - & $12.4(9.5)$ & - \\
\hline During post-index period until switch to other LAI & $12.7(10.6)$ & - & $12.4(9.5)$ & - \\
\hline \multicolumn{5}{|c|}{ Number of injections of initial agent during post-index period until switch to other LAI, $n$ (\%) } \\
\hline 1 & $92(8.1)$ & - & $38(7.1)$ & - \\
\hline 2 & $56(4.9)$ & - & $26(4.9)$ & - \\
\hline 3 & $43(3.8)$ & - & $17(3.2)$ & - \\
\hline $4-6$ & $209(18.5)$ & - & $106(19.9)$ & - \\
\hline $7-10$ & $150(13.3)$ & - & $84(15.8)$ & - \\
\hline $11-20$ & $389(34.4)$ & - & $175(32.8)$ & - \\
\hline $21+$ & $193(17.0)$ & - & $87(16.3)$ & - \\
\hline \multicolumn{5}{|l|}{ Number of prescriptions of initial agent, mean (SD) } \\
\hline During post-index period & $5.6(4.5)$ & $4.0(3.3)$ & $5.5(4.5)$ & $5.0(3.3)$ \\
\hline During post-index period until switch to other LAI & $5.6(4.5)$ & $4.0(3.3)$ & $5.5(4.5)$ & $5.0(3.3)$ \\
\hline \multicolumn{5}{|c|}{ Number of prescriptions of initial agent during post-index period until switch to other LAI, $n$ (\%) } \\
\hline 1 & $247(21.8)$ & $516(34.0)$ & $115(21.6)$ & $49(21.3)$ \\
\hline 2 & $133(11.7)$ & $205(13.5)$ & $67(12.6)$ & $21(9.1)$ \\
\hline 3 & $104(9.2)$ & $119(7.8)$ & $53(9.9)$ & $17(7.4)$ \\
\hline $4-6$ & $279(24.7)$ & $344(22.7)$ & $126(23.6)$ & $75(32.6)$ \\
\hline $7-10$ & $169(14.9)$ & $272(17.9)$ & $83(15.6)$ & $57(24.8)$ \\
\hline $11-20$ & $193(17.0)$ & $60(4.0)$ & $87(16.3)$ & $10(4.3)$ \\
\hline $21+$ & $7(0.6)$ & $2(0.1)$ & $2(0.4)$ & $1(0.4)$ \\
\hline $\begin{array}{l}\text { Number of patients with at least one prescription during pre-index } \\
\text { period, } n(\%)\end{array}$ & $809(71.5)$ & $1518(100.0)$ & $278(52.2)$ & $230(100.0)$ \\
\hline \multicolumn{5}{|l|}{ Last prescribed agent during pre-index period, $n(\%)$} \\
\hline Levomepromazine & $12(1.1)$ & $14(0.9)$ & $4(0.8)$ & $3(1.3)$ \\
\hline Fluphenazine & $6(0.5)$ & $17(1.1)$ & $2(0.4)$ & $5(2.2)$ \\
\hline Perphenazine & $4(0.4)$ & $8(0.5)$ & $3(0.6)$ & $4(1.7)$ \\
\hline Perazine & $15(1.3)$ & $30(2.0)$ & $3(0.6)$ & $8(3.5)$ \\
\hline Haloperidol & $36(3.2)$ & 44 (2.9) & $12(2.3)$ & $12(5.2)$ \\
\hline Melperone & $13(1.1)$ & $17(1.1)$ & $5(0.9)$ & $2(0.9)$ \\
\hline Pipamperone & $33(2.9)$ & $30(2.0)$ & $9(1.7)$ & $6(2.6)$ \\
\hline Benperidol & $4(0.4)$ & $15(1.0)$ & $1(0.2)$ & $1(0.4)$ \\
\hline Ziprasidone & $9(0.8)$ & $56(3.7)$ & $4(0.8)$ & $7(3.0)$ \\
\hline Flupentixol & $20(1.8)$ & $45(3.0)$ & $8(1.5)$ & $6(2.6)$ \\
\hline Chlorprothixene & $28(2.5)$ & $31(2.0)$ & $5(0.9)$ & $4(1.7)$ \\
\hline Pimozide & $1(0.1)$ & $9(0.6)$ & $0(0.0)$ & $4(1.7)$ \\
\hline Clozapine & $42(3.7)$ & $199(13.1)$ & $11(2.1)$ & $10(4.3)$ \\
\hline Olanzapine & $79(7.0)$ & $212(14.0)$ & $29(5.4)$ & $29(12.6)$ \\
\hline Quetiapine & $120(10.6)$ & $237(15.6)$ & $40(7.5)$ & $35(15.2)$ \\
\hline Sulpiride & $4(0.4)$ & $14(0.9)$ & $3(0.6)$ & $4(1.7)$ \\
\hline Amisulpride & $53(4.7)$ & $151(9.9)$ & $23(4.3)$ & $18(7.8)$ \\
\hline Lithium & $11(1.0)$ & $30(2.0)$ & $2(0.4)$ & $6(2.6)$ \\
\hline Prothipendyl & $11(1.0)$ & $17(1.1)$ & $3(0.6)$ & $1(0.4)$ \\
\hline Risperidone & $213(18.8)$ & 209 (13.8) & $72(13.5)$ & $47(20.4)$ \\
\hline Aripiprazole & $84(7.4)$ & $113(7.4)$ & $36(6.8)$ & $13(5.7)$ \\
\hline
\end{tabular}


Table 2 (continued)

\begin{tabular}{|c|c|c|c|c|}
\hline \multirow[t]{2}{*}{ Parameter } & \multicolumn{2}{|l|}{ Overall cohort } & \multicolumn{2}{|c|}{ Complete switch cohort } \\
\hline & LAI $(n=1,132)$ & Oral $(n=1,518)$ & LAI $(n=533)$ & Oral $(n=230)$ \\
\hline \multicolumn{5}{|c|}{ Agents used during pre-index period, $n(\%)$} \\
\hline Levomepromazine & $31(2.7)$ & $46(3.0)$ & $6(1.1)$ & $6(2.6)$ \\
\hline Fluphenazine & $12(1.1)$ & $26(1.7)$ & $2(0.4)$ & $5(2.2)$ \\
\hline Perphenazine & $6(0.5)$ & $14(0.9)$ & $3(0.6)$ & $4(1.7)$ \\
\hline Perazine & $34(3.0)$ & $58(3.8)$ & $5(0.9)$ & $12(5.2)$ \\
\hline Thioridazine & $1(0.1)$ & $9(0.6)$ & $0(0.0)$ & $3(1.3)$ \\
\hline Haloperidol & $74(6.5)$ & $76(5.0)$ & $21(3.9)$ & $16(7.0)$ \\
\hline Melperone & $40(3.5)$ & $45(3.0)$ & $14(2.6)$ & $3(1.3)$ \\
\hline Pipamperone & $79(7.0)$ & $62(4.1)$ & $23(4.3)$ & $9(3.9)$ \\
\hline Benperidol & $12(1.1)$ & $21(1.4)$ & $3(0.6)$ & $3(1.3)$ \\
\hline Ziprasidone & $29(2.6)$ & 75 (4.9) & $9(1.7)$ & $7(3.0)$ \\
\hline Flupentixol & $51(4.5)$ & $72(4.7)$ & $17(3.2)$ & $12(5.2)$ \\
\hline Chlorprothixene & $73(6.4)$ & $79(5.2)$ & $15(2.8)$ & $7(3.0)$ \\
\hline Zuclopenthixol & $24(2.1)$ & $14(0.9)$ & $6(1.1)$ & $1(0.4)$ \\
\hline Pimozide & $3(0.3)$ & $11(0.7)$ & $2(0.4)$ & $5(2.2)$ \\
\hline Clozapine & $72(6.4)$ & $236(15.5)$ & $14(2.6)$ & $13(5.7)$ \\
\hline Olanzapine & $181(16.0)$ & 287 (18.9) & $51(9.6)$ & $30(13.0)$ \\
\hline Quetiapine & $249(22.0)$ & $341(22.5)$ & $58(10.9)$ & $42(18.3)$ \\
\hline Sulpiride & $7(0.6)$ & $23(1.5)$ & $3(0.6)$ & $5(2.2)$ \\
\hline Amisulpride & $99(8.7)$ & $223(14.7)$ & 37 (6.9) & $25(10.9)$ \\
\hline Lithium & $26(2.3)$ & $56(3.7)$ & $2(0.4)$ & $6(2.6)$ \\
\hline Prothipendyl & $30(2.7)$ & $47(3.1)$ & $4(0.8)$ & $3(1.3)$ \\
\hline Risperidone & 304 (26.9) & $281(18.5)$ & 98 (18.4) & $58(25.2)$ \\
\hline Aripiprazole & $178(15.7)$ & $183(12.1)$ & $53(9.9)$ & $19(8.3)$ \\
\hline \multicolumn{5}{|c|}{ Agents used during post-index period, $n(\%)$} \\
\hline Levomepromazine & $40(3.5)$ & $102(6.7)$ & $9(1.7)$ & $8(3.5)$ \\
\hline Fluphenazine & $47(4.2)$ & $17(1.1)$ & $20(3.8)$ & $0(0.0)$ \\
\hline Perazine & $44(3.9)$ & $104(6.9)$ & $8(1.5)$ & $13(5.7)$ \\
\hline Haloperidol & $138(12.2)$ & $148(9.7)$ & $49(9.2)$ & $18(7.8)$ \\
\hline Melperone & $53(4.7)$ & $186(12.3)$ & $16(3.0)$ & $10(4.3)$ \\
\hline Pipamperone & $99(8.7)$ & $264(17.4)$ & $15(2.8)$ & $17(7.4)$ \\
\hline Benperidol & $16(1.4)$ & $26(1.7)$ & $0(0.0)$ & $3(1.3)$ \\
\hline Ziprasidone & $23(2.0)$ & $106(7.0)$ & $4(0.8)$ & $10(4.3)$ \\
\hline Flupentixol & $155(13.7)$ & $110(7.2)$ & $60(11.3)$ & $7(3.0)$ \\
\hline Chlorprothixene & $103(9.1)$ & $196(12.9)$ & $21(3.9)$ & $11(4.8)$ \\
\hline Zuclopenthixol & $52(4.6)$ & $22(1.4)$ & $18(3.4)$ & $1(0.4)$ \\
\hline Fluspirilene & $105(9.3)$ & $0(0.0)$ & $59(11.1)$ & $0(0.0)$ \\
\hline Clozapine & 78 (6.9) & $255(16.8)$ & $15(2.8)$ & $14(6.1)$ \\
\hline Olanzapine & 195 (17.2) & $396(26.1)$ & $59(11.1)$ & $51(22.2)$ \\
\hline Quetiapine & $255(22.5)$ & 555 (36.6) & $49(9.2)$ & $74(32.2)$ \\
\hline Sulpiride & $9(0.8)$ & $22(1.4)$ & $3(0.6)$ & $2(0.9)$ \\
\hline Tiapride & $5(0.4)$ & $15(1.0)$ & $2(0.4)$ & $2(0.9)$ \\
\hline Amisulpride & $58(5.1)$ & $311(20.5)$ & $10(1.9)$ & $38(16.5)$ \\
\hline Lithium & $36(3.2)$ & $69(4.5)$ & $4(0.8)$ & $8(3.5)$ \\
\hline Prothipendyl & $50(4.4)$ & $161(10.6)$ & $10(1.9)$ & $13(5.7)$ \\
\hline Risperidone & $417(36.8)$ & $412(27.1)$ & $154(28.9)$ & $48(20.9)$ \\
\hline Aripiprazole & $255(22.5)$ & $438(28.9)$ & $103(19.3)$ & $51(22.2)$ \\
\hline Paliperidone & $413(36.5)$ & $0(0.0)$ & $189(35.5)$ & $0(0.0)$ \\
\hline
\end{tabular}


Table 2 (continued)

\begin{tabular}{|c|c|c|c|c|}
\hline \multirow[t]{2}{*}{ Parameter } & \multicolumn{2}{|l|}{ Overall cohort } & \multicolumn{2}{|c|}{ Complete switch cohort } \\
\hline & LAI $(n=1,132)$ & Oral $(n=1,518)$ & LAI $(n=533)$ & Oral $(n=230)$ \\
\hline \multicolumn{5}{|c|}{ LAI used during post-index period, $n(\%)$} \\
\hline Fluphenazine & $42(3.7)$ & $0(0.0)$ & $18(3.4)$ & $0(0.0)$ \\
\hline Haloperidol & $85(7.5)$ & $0(0.0)$ & $40(7.5)$ & $0(0.0)$ \\
\hline Flupentixol & $129(11.4)$ & $0(0.0)$ & $53(9.9)$ & $0(0.0)$ \\
\hline Zuclopenthixol & $30(2.7)$ & $0(0.0)$ & $14(2.6)$ & $0(0.0)$ \\
\hline Fluspirilene & $105(9.3)$ & $0(0.0)$ & $59(11.1)$ & $0(0.0)$ \\
\hline Olanzapine & $39(3.4)$ & $0(0.0)$ & $27(5.1)$ & $0(0.0)$ \\
\hline Risperidone & $280(24.7)$ & $1(0.1)$ & $125(23.5)$ & $0(0.0)$ \\
\hline Aripiprazole & $124(11.0)$ & $0(0.0)$ & $56(10.5)$ & $0(0.0)$ \\
\hline Paliperidone & $413(36.5)$ & $0(0.0)$ & $189(35.5)$ & $0(0.0)$ \\
\hline
\end{tabular}

Medications that were used $<1 \%$ in any group were not added in the table but are listed here as per the parameter. In last prescribed agent during pre-index period - thioridazine, bromperidol, sertindole, zuclopenthixol, fluspirilene, asenapine, tiapride, zotepine, and paliperidone; in agents used during pre-index period-bromperidol, sertindole, fluspirilene, asenapine, tiapride, zotepine, and paliperidone; in agents used during post-index period-perphenazine, thioridazine, bromperidol, sertindole, pimozide, asenapine, and zotepine; and in LAI during post-index period-perphenazine

$L A I$ long-acting injectable antipsychotic, $S D$ standard deviation

Table 3 Adherence and persistence indicators

\begin{tabular}{|c|c|c|c|c|}
\hline \multirow[t]{2}{*}{ Parameter } & \multicolumn{2}{|l|}{ Overall cohort } & \multicolumn{2}{|c|}{ Complete switch cohort } \\
\hline & LAI $(n=1,132)$ & Oral $(n=1,518)$ & LAI $(n=533)$ & Oral $(n=230)$ \\
\hline \multicolumn{5}{|l|}{ PDC } \\
\hline Mean (SD) & $0.6(0.3)$ & $0.3(0.3)$ & $0.6(0.3)$ & $0.4(0.3)$ \\
\hline Median (IQR) & $0.6(0.2-0.9)$ & $0.1(0.0-0.5)$ & $0.6(0.2-0.9)$ & $0.4(0.1-0.8)$ \\
\hline \multicolumn{5}{|c|}{ Adherence, $n(\%)$} \\
\hline $\mathrm{PDC} \geq 0.8$ & $401(35.4)$ & $176(11.6)$ & $176(33.0)$ & $50(21.7)$ \\
\hline \multicolumn{5}{|c|}{ Persistence, $n(\%)$} \\
\hline No 30-day gap & $299(26.4)$ & $145(9.6)$ & $138(25.9)$ & $43(18.7)$ \\
\hline No 60-day gap & $461(40.7)$ & $300(19.8)$ & $203(38.1)$ & $76(33.0)$ \\
\hline No 90-day gap & $546(48.2)$ & $430(28.3)$ & $245(46.0)$ & $98(42.6)$ \\
\hline
\end{tabular}

$I Q R$ interquartile range, $L A I$ long acting injectable antipsychotic, $P D C$ proportion of days covered, $S D$ standard deviation

\section{Discussion}

This retrospective study based on German health-claims data analyzed antipsychotic medication adherence, persistence, and discontinuation among patients with schizophrenia who initiated an LAI and those who switched their OAP regimen. LAI users had better medication adherence $(35.4 \%$ vs. $11.6 \%$ ) and better persistence (no 30 -day gap: $26.4 \%$ vs. $9.6 \%$, no 60 -day gap: $40.7 \%$ vs. $19.8 \%$, and no 90 -day gap: $48.2 \%$ vs. $28.3 \%$ ) during the 1 -year post-index period, as well as a 166 days longer median time to discontinuation of antipsychotic treatment compared to OAP users. Sensitivity analysis also showed greater adherence and persistence and a 91.5 days longer median time to discontinuation of antipsychotic treatment among LAI users compared to OAP users.
However, in this analysis the results of the Cox regression indicate only numerical superiority of LAIs.

Similar German studies comparing persistence of LAIs and OAPs are unavailable. However, our results are in line with observational studies of varying sample sizes conducted in the USA [20, 21, 25, 29, 43]. Greene et al. [20] analyzed Medicaid data and reported that a greater proportion of LAI users than OAP users had PDC $\geq 0.8$ after a year of follow-up (33.9\% vs. $25.5 \% ; p<0.001)$. Moreover, the discontinuation rate was lower among LAI users compared with OAP users $(63.2 \%$ vs. $72.0 \%$; $p<0.001)$. Another study using Medicaid data reported SGA LAI users to be more adherent than OAP users to their prescribed index medication after a year of follow-up $(27.2 \%$ vs. $24.6 \%$; $p<0.05)$ [43]. In addition, a higher persistence rate (no 60-day gap) among SGA LAI users was reported (37.1\% vs. $30.2 \%)$. 
Fig. 2 Unadjusted KaplanMeier curve for overall cohort (LAIs vs. oral). LAI long-acting injectable antipsychotic

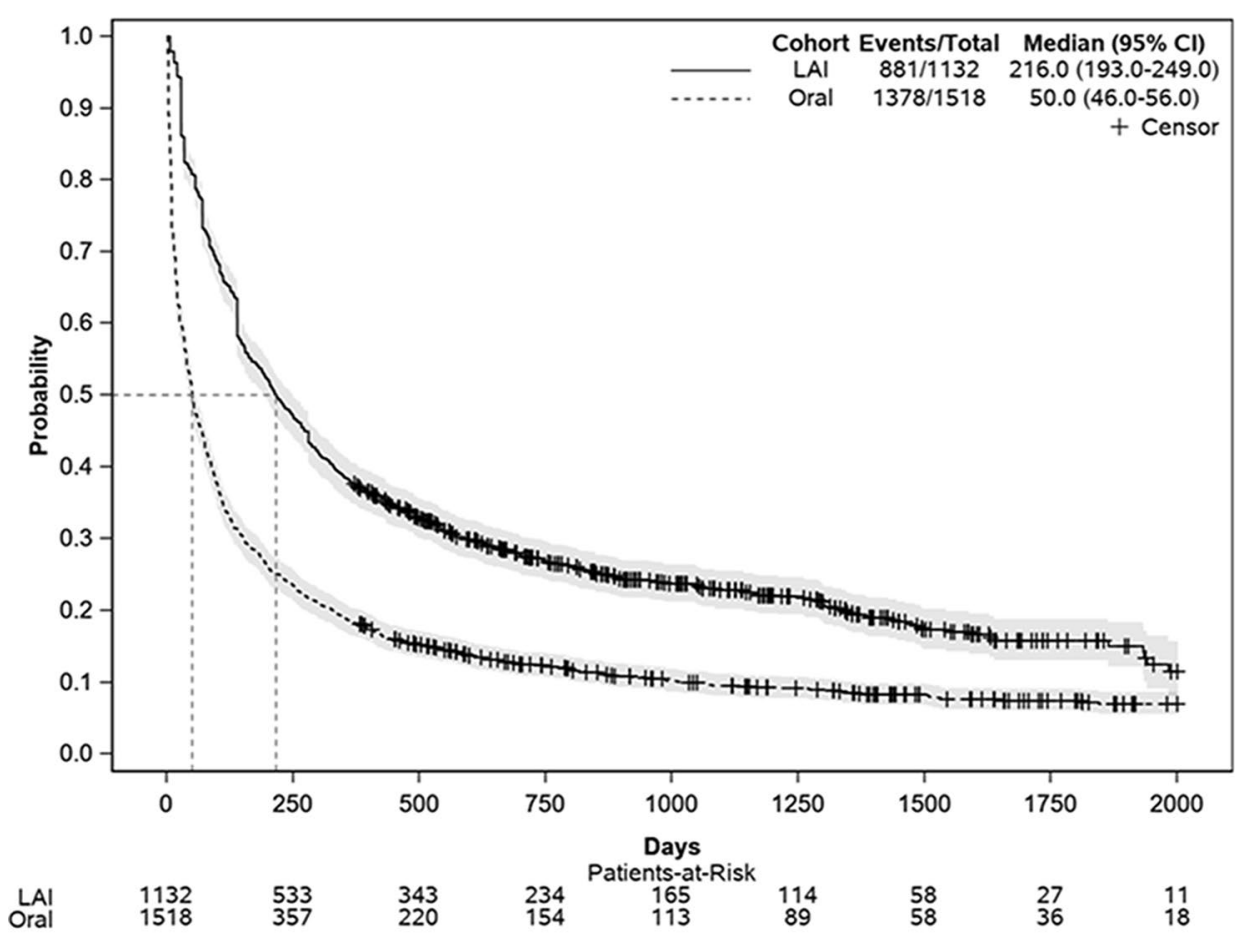

Table 4 Time to end of therapy

\begin{tabular}{|c|c|c|c|c|}
\hline \multirow[t]{2}{*}{ Parameter } & \multicolumn{2}{|l|}{ Overall cohort } & \multicolumn{2}{|c|}{ Complete switch cohort } \\
\hline & LAI $(n=1,132)$ & Oral $(n=1,518)$ & LAI $(n=533)$ & Oral $(n=230)$ \\
\hline \multicolumn{5}{|l|}{ Summary } \\
\hline End of therapy (n) & 881 & 1378 & 420 & 190 \\
\hline Censored, $n(\%)$ & $251(22.2)$ & $140(9.2)$ & $113(21.2)$ & $40(17.4)$ \\
\hline \multicolumn{5}{|l|}{ Time to end of therapy (days) } \\
\hline Mean (SE) & $580.6(22)$ & $277(13.5)$ & $558.4(31.9)$ & $377.1(32.8)$ \\
\hline Median $(95 \%$ CI) & $216(193-249)$ & $50(46-56)$ & $211(163-247)$ & $119.5(86-157)$ \\
\hline \multicolumn{5}{|c|}{ Reason for end of therapy among patients whose therapy ended, $n(\%)$} \\
\hline Gap $>60$ days & $518(58.8)$ & $543(39.4)$ & $317(75.5)$ & $144(75.8)$ \\
\hline $\begin{array}{l}\text { Other agent taken after last } \\
\text { prescription }\end{array}$ & $363(41.2)$ & $835(60.6)$ & $103(24.5)$ & $46(24.2)$ \\
\hline
\end{tabular}

$C I$ confidence interval, $L A I$ long-acting injectable, $S E$ standard error

Previous research has also demonstrated the greater risk of antipsychotic treatment discontinuation among OAP users. Greene et al. [20] reported a $20 \%$ greater risk of antipsychotic discontinuation among OAP users (HR 1.20; 95\% CI 1.13-1.28; $p<0.001$ ), and Pilon et al. [43] reported $46 \%$ greater odds of persistence among SGA LAI users compared with OAP users (OR 1.46; 95\% CI 1.32-1.62; $p<0.001$ ). Paliperidone palmitate LAI users were observed to have the greatest odds of persistence compared with OAP users (OR $1.53 ; 95 \%$ CI $1.39-1.69 ; p<0.001)$ [25].

In the current study, usage of OAP and greater CCI were associated with increased risk of antipsychotic treatment discontinuation. These results are consistent with previous research. Verdoux et al. [44] compared propensity scorematched French patients with schizophrenia who were initiated on LAIs or OAPs and reported greater treatment discontinuation among OAP users $(69 \%)$ versus LAI users (57\%) (adjusted relative risk 1.6; 95\% CI 1.23-2.07). Greene et al. [20] also reported a $20 \%$ greater risk of antipsychotic discontinuation among OAP users compared with LAI users. In the current study, we observed a high proportion of LAI and OAP users suffering from comorbid chronic pulmonary disease and diabetes. These patients may have concomitant prescriptions along with their antipsychotic medication. 
Table 5 Risk of discontinuation of treatment

\begin{tabular}{|c|c|c|c|c|}
\hline \multirow[t]{2}{*}{ Variable } & \multicolumn{2}{|l|}{ Overall cohort } & \multicolumn{2}{|c|}{ Complete switch cohort } \\
\hline & $\mathrm{HR}(95 \% \mathrm{CI})$ & $p$ value (Wald test) & HR $(95 \%$ CI $)$ & $\begin{array}{l}p \text { value } \\
\text { (Wald } \\
\text { test) }\end{array}$ \\
\hline \multicolumn{5}{|l|}{ Model 1} \\
\hline \multicolumn{5}{|l|}{ Medication (reference LAI) } \\
\hline Oral & $1.89(1.73-2.06)$ & $<0.001$ & $1.19(1.00-1.42)$ & 0.050 \\
\hline Age & $1.00(0.99-1.00)$ & 0.226 & $0.99(0.99-1.00)$ & 0.100 \\
\hline \multicolumn{5}{|l|}{ Sex (reference men) } \\
\hline Women & $1.12(0.94-1.33)$ & 0.208 & $1.17(0.84-1.63)$ & 0.359 \\
\hline CCI & $1.04(1.00-1.07)$ & 0.023 & $1.09(1.02-1.16)$ & 0.007 \\
\hline \multicolumn{5}{|l|}{ Model 2} \\
\hline \multicolumn{5}{|l|}{ Medication (reference LAI) } \\
\hline Oral & $1.87(1.70-2.05)$ & $<0.001$ & $1.08(0.89-1.31)$ & 0.453 \\
\hline Age & $1.00(0.99-1.00)$ & 0.124 & $0.99(0.99-1.00)$ & 0.163 \\
\hline \multicolumn{5}{|l|}{ Sex (reference men) } \\
\hline Women & $1.14(0.96-1.36)$ & 0.136 & $1.25(0.88-1.76)$ & 0.210 \\
\hline CCI & $1.04(1.00-1.08)$ & 0.026 & $1.08(1.01-1.15)$ & 0.032 \\
\hline \multicolumn{5}{|c|}{ Insurance status (reference retired person) } \\
\hline No compulsory insurance & $1.18(0.65-2.15)$ & 0.582 & $1.48(0.37-5.99)$ & 0.581 \\
\hline Compulsory insurance & $0.94(0.83-1.05)$ & 0.258 & $1.06(0.85-1.34)$ & 0.603 \\
\hline Voluntary insurance & $0.97(0.83-1.13)$ & 0.676 & $1.06(0.76-1.48)$ & 0.720 \\
\hline Unemployed person & $1.07(0.92-1.24)$ & 0.397 & $1.14(0.87-1.49)$ & 0.346 \\
\hline Pension claimant & $0.44(0.21-0.92)$ & 0.030 & $0.35(0.09-1.41)$ & 0.138 \\
\hline Special group of persons & $1.15(0.79-1.67)$ & 0.460 & $1.09(0.48-2.47)$ & 0.835 \\
\hline Family member & $0.86(0.71-1.04)$ & 0.110 & $0.78(0.52-1.16)$ & 0.214 \\
\hline Unknown & $0.99(0.84-1.17)$ & 0.938 & $1.09(0.79-1.50)$ & 0.614 \\
\hline Medication costs (pre-index) ${ }^{a}$ & $0.99(0.97-1.01)$ & 0.395 & $1.02(0.94-1.11)$ & 0.606 \\
\hline Visit costs (pre-index) ${ }^{a}$ & $1.02(0.99-1.05)$ & 0.258 & $1.12(0.98-1.29)$ & 0.105 \\
\hline $\begin{array}{l}\text { Psychiatric inpatient stay costs (pre- } \\
\text { index) }^{\mathrm{a}}\end{array}$ & $1.00(0.99-1.00)$ & 0.070 & $0.99(0.98-1.00)$ & 0.014 \\
\hline
\end{tabular}

AIC for model 1 is 32,189 for overall cohort and 7313 for complete switch cohort. AIC for model 2 is 32,194 for overall cohort and 7318 for complete switch cohort

In insurance status, although pension claimant is seen to significantly affect the risk of treatment discontinuation, we have not considered it. In Germany, a 'pension claimant' is defined as a person who has applied to receive pension. Once the application is successful, the status of the person changes to 'retired', which was the reference category for the analysis

AIC Akaike Information Criterion, CI confidence interval, LAI long-acting injectable

${ }^{\mathrm{a}}$ Costs transformed to $€ 1000$

However, as patients with such conditions are often nonadherent [45], it is likely that their antipsychotic prescriptions were also not adhered to, resulting in the observed greater risk of antipsychotic treatment discontinuation. We did not find factors such as young age to be significantly associated with a higher risk of antipsychotic treatment discontinuation. However, a systematic literature review found younger age (especially $<35$ years) to be a significant risk factor for non-adherence to antipsychotics [46]. We have also made similar observations during routine clinical practice, as young patients often fail to grasp the severity of their condition and do not adhere to the prescribed treatment regimen.

Multiple factors have been associated with non-adherence to medication among patients with schizophrenia [16, 47]. Among these factors, lack of insight/awareness of illness, negative attitude towards medication, fear of social stigma if others observe the patient taking medication, and treatment complexity are the key determinants of non-adherence. Although LAIs cannot resolve all the factors associated with non-adherence, a few issues can be tackled. LAIs have a simple treatment regimen (e.g., an injection once every month or every 3 months), are administered in a safe 
Fig. 3 Unadjusted KaplanMeier curve for complete switch cohort (LAIs vs. oral). LAI long-acting injectable antipsychotic

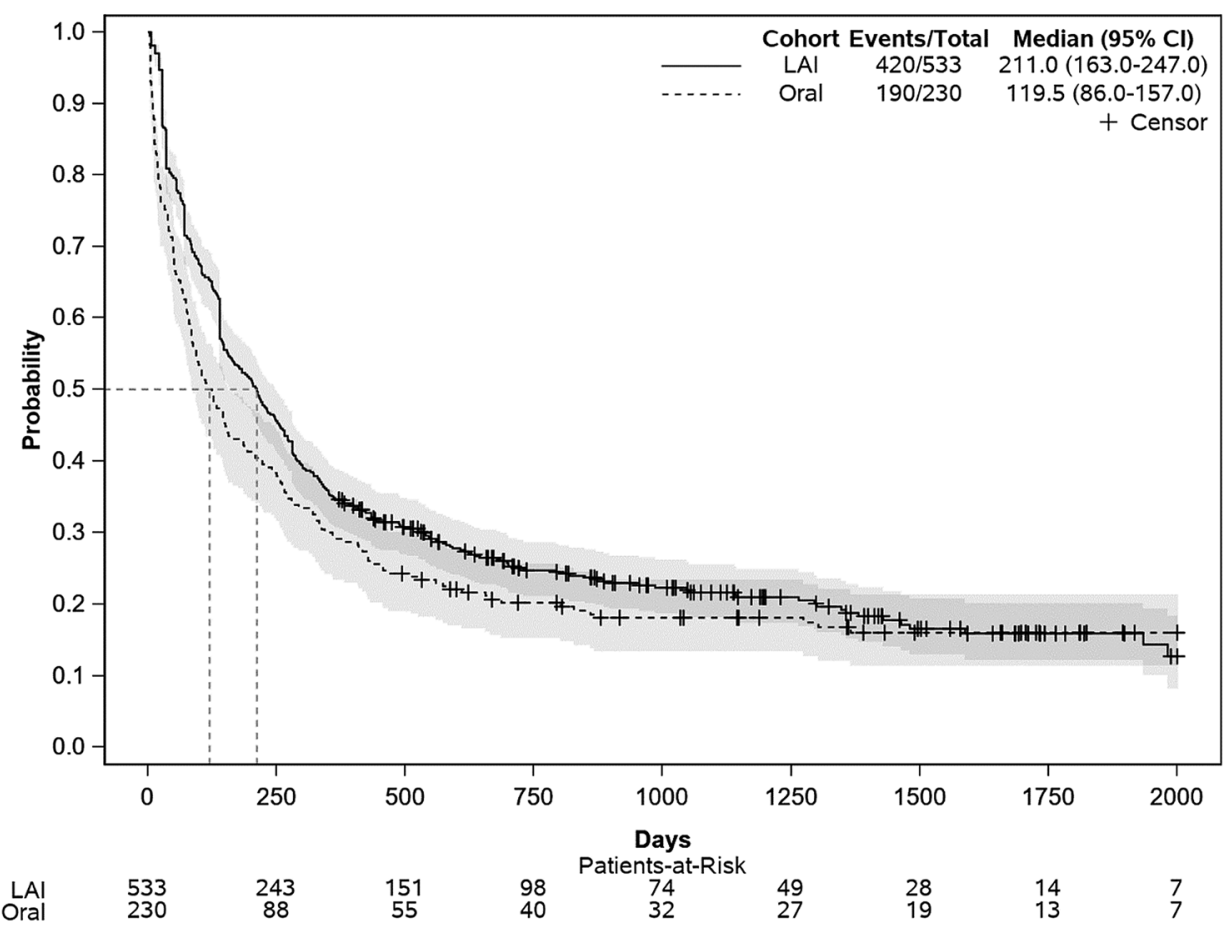

space (i.e., clinic), and have a prolonged therapeutic action, which result in better adherence and longer time to treatment discontinuation.

Poor adherence among patients with schizophrenia is associated with increased risk of rehospitalization [48]; hence, increasing adherence through LAI usage is necessary. Previous research has shown a reduction in risk of hospitalization as well as treatment costs with LAI usage in Germany [49]. Furthermore, as caregivers of patients with schizophrenia are negatively affected [50], better functioning patients might ease the caregivers' burden. Sruamsiri et al. [50] reported lower productivity losses among caregivers whose relatives were on LAIs, although the sample size was too small to reveal any significant differences. LAI use could also reduce the economic burden for payors as reported by Mahlich et al. [51], who modeled the costs incurred by the Japanese healthcare system and reported significant cost savings of $>$ US\$68 million on introduction of paliperidone palmitate LAI.

Despite the benefits associated with LAIs, they are often prescribed only to non-adherent patients, and patients with first-episode schizophrenia are hardly considered suitable candidates [52], possibly due to budget constraints. In Germany, the market share of LAIs in terms of patients under treatment is around $12 \%$ according to IQVIA market research data. Clinician-related barriers such as lack of knowledge, mistaken belief about greater adverse effects on using LAIs, and lack of resources, as well as patient-related barriers such as stigma, fear of injection, injection site pain, and cost, negatively impact the uptake of LAIs in clinical practice [53]. However, as evidence supporting the benefits of LAIs are available, approaches to train clinicians and their staff as well as educate patients are required.

\subsection{Limitations}

There are a few limitations of the present study. Due to the inherent nature of health-claims data, it is possible that a diagnosis of schizophrenia was a result of misclassification or coding errors. Claims data also insufficiently reflect disease severity and other influencing circumstances. Although claims data were used to identify antipsychotic utilization, we can only presume that the medication was consumed, as the data only support receipt and payment. Nevertheless, since LAIs are generally administered by a healthcare professional, a claim for an LAI provides confidence that the effective dose was administered to the patient. We assessed the persistence of LAIs as a whole and not by generation, thus missing out on describing the increased adherence and persistence among SGAs that were observed by Pilon et al. [43]. Previous research has shown the superiority of SGAs compared with first-generation antipsychotics in terms of adherence and persistence $[25,43]$ in the USA. Similar studies could be designed for Germany too. Finally, we observed crossing Kaplan-Meier curves after 1500 days in our sensitivity analysis, which is a violation of the proportional hazard assumption. As the number of patients at risk at that time was low, it is unlikely that this had a major effect on our results. 


\section{Conclusion}

This study analyzed a large health-claims database and found LAIs to be associated with increased adherence and persistence as well as with reduction of antipsychotic treatment discontinuation. OAP usage and greater CCI were associated with increased risk of discontinuation. Future research could evaluate the long-term effect of LAIs on similar parameters as well as delineate the effect of individual antipsychotics.

Acknowledgements We thank Leo J. Philip Tharappel from Siro Clinpharm Pvt Ltd. For providing medical writing and editorial assistance. Open Access funding enabled and organized by Projekt DEAL.

\section{Declarations}

Funding The study was funded by Janssen-Cilag, Neuss, Germany.

Conflict of interest Jörg Mahlich, Kerstin Olbrich, and Antonie Wimmer are employees of Janssen-Cilag and may have stock/stock options. Adrian Wilk is an employee of Team Gesundheit GmbH, which received funding for this study from Janssen-Cilag. Claus Wolff-Menzler has no conflict of interest to declare.

Ethics approval All procedures were in accordance with the 1964 Helsinki declaration and its later amendments or comparable ethical standards. Approval of a local ethics board was not required as this study used anonymized German claims data.

Informed consent Not applicable.

Open Access This article is licensed under a Creative Commons Attribution-NonCommercial 4.0 International License, which permits any non-commercial use, sharing, adaptation, distribution and reproduction in any medium or format, as long as you give appropriate credit to the original author(s) and the source, provide a link to the Creative Commons licence, and indicate if changes were made. The images or other third party material in this article are included in the article's Creative Commons licence, unless indicated otherwise in a credit line to the material. If material is not included in the article's Creative Commons licence and your intended use is not permitted by statutory regulation or exceeds the permitted use, you will need to obtain permission directly from the copyright holder. To view a copy of this licence, visit http://creativecommons.org/licenses/by-nc/4.0/.

\section{References}

1. Kern EF, Maney M, Miller DR, et al. Failure of ICD-9-CM codes to identify patients with comorbid chronic kidney disease in diabetes. Health Serv Res. 2006;41:564-80.

2. Gaebel W, Wölwer W. Gesundheitsberichterstattung des Bundes: Schizoprenie. Berlin: Robert Koch Institut; 2010.

3. Fioravanti M, Bianchi V, Cinti ME. Cognitive deficits in schizophrenia: an updated metanalysis of the scientific evidence. BMC Psychiatry. 2012;12:64.

4. Kitchen H, Rofail D, Heron L, et al. Cognitive impairment associated with schizophrenia: a review of the humanistic burden. Adv Ther. 2012;29:148-62.
5. Frey S. The economic burden of schizophrenia in Germany: a population-based retrospective cohort study using genetic matching. Eur Psychiatry. 2014;29:479-89.

6. Kane JM, Correll CU. Pharmacologic treatment of schizophrenia. Dialogues Clin Neurosci. 2010;12:345-57.

7. Barkhof E, Meijer CJ, de Sonneville LM, et al. Interventions to improve adherence to antipsychotic medication in patients with schizophrenia-a review of the past decade. Eur Psychiatry. 2012;27:9-18.

8. Rummel-Kluge C, Schuster T, Peters S, et al. Partial compliance with antipsychotic medication is common in patients with schizophrenia. Aust N Z J Psychiatry. 2008;42:382-8.

9. Marder SR. Overview of partial compliance. J Clin Psychiatry. 2003;64(Suppl 16):3-9.

10. Lang K, Meyers JL, Korn JR, et al. Medication adherence and hospitalization among patients with schizophrenia treated with antipsychotics. Psychiatr Serv. 2010;61:1239-47.

11. Eaddy M, Grogg A, Locklear J. Assessment of compliance with antipsychotic treatment and resource utilization in a Medicaid population. Clin Ther. 2005;27:263-72.

12. Kozma CM, Weiden PJ. Partial compliance with antipsychotics increases mental health hospitalizations in schizophrenic patients: analysis of a national managed care database. Am Health Drug Benefits. 2009;2:31-8.

13. Gilmer TP, Dolder CR, Lacro JP, et al. Adherence to treatment with antipsychotic medication and health care costs among Medicaid beneficiaries with schizophrenia. Am J Psychiatry. 2004:161:692-9.

14. Morken G, Widen JH, Grawe RW. Non-adherence to antipsychotic medication, relapse and rehospitalisation in recent-onset schizophrenia. BMC Psychiatry. 2008;8:32.

15. Knapp M, King D, Pugner K, et al. Non-adherence to antipsychotic medication regimens: associations with resource use and costs. Br J Psychiatry. 2004;184:509-16.

16. Higashi K, Medic G, Littlewood KJ, et al. Medication adherence in schizophrenia: factors influencing adherence and consequences of nonadherence, a systematic literature review. Ther Adv Psychopharmacol. 2013;3:200-18.

17. Willis M, Svensson M, Lothgren M, et al. The impact on schizophrenia-related hospital utilization and costs of switching to long-acting risperidone injections in Sweden. Eur J Health Econ. 2010;11:585-94.

18. Tiihonen J, Haukka J, Taylor M, et al. A nationwide cohort study of oral and depot antipsychotics after first hospitalization for schizophrenia. Am J Psychiatry. 2011;168:603-9.

19. Grimaldi-Bensouda L, Rouillon F, Astruc B, et al. Does longacting injectable risperidone make a difference to the real-life treatment of schizophrenia? Results of the Cohort for the General study of Schizophrenia (CGS). Schizophr Res. 2012;134:187-94.

20. Greene M, Yan T, Chang E, et al. Medication adherence and discontinuation of long-acting injectable versus oral antipsychotics in patients with schizophrenia or bipolar disorder. J Med Econ. 2018;21:127-34.

21. Marcus SC, Zummo J, Pettit AR, et al. Antipsychotic adherence and rehospitalization in schizophrenia patients receiving oral versus long-acting injectable antipsychotics following hospital discharge. J Manag Care Spec Pharm. 2015;21:754-68.

22. Thiem H, Folkerts H, Völkel L. Short-acting antipsychotics or long-acting injectables? A treatment comparison in patients with schizophrenia [Kurzwirksame Antipsychotika oder DepotInjektionen? Ein Vergleich der Behandlung von Patienten mit Schizophrenie]. Gesundheitsökonomie \& Qualitätsmanagement. 2020;25:170-8.

23. Potempa C, Rychlik R. Hospitalization rates and resource utilization of schizophrenic patients switched from oral antipsychotics to aripiprazole-depot in Germany. Health Econ Rev. 2018;8:30. 
24. Lin J, Wong B, Offord S, et al. Healthcare cost reductions associated with the use of LAI formulations of antipsychotic medications versus oral among patients with schizophrenia. J Behav Health Serv Res. 2013;40:355-66.

25. Pilon D, Tandon N, Lafeuille MH, et al. Treatment patterns, health care resource utilization, and spending in Medicaid beneficiaries initiating second-generation long-acting injectable agents versus oral atypical antipsychotics. Clin Ther. 2017;39(1972-1985):e1972.

26. Zeidler J, Mahlich J, Greiner W, et al. Cost effectiveness of paliperidone palmitate for the treatment of schizophrenia in Germany. Appl Health Econ Health Policy. 2013;11:509-21.

27. Laux G, Heeg B, van Hout BA, et al. Costs and effects of longacting risperidone compared with oral atypical and conventional depot formulations in Germany. Pharmacoeconomics. 2005;23(Suppl 1):49-61.

28. Achilla E, McCrone P. The cost effectiveness of long-acting/ extended-release antipsychotics for the treatment of schizophrenia: a systematic review of economic evaluations. Appl Health Econ Health Policy. 2013;11:95-106.

29. Joshi K, Muser E, Xu Y, et al. Adherence and economic impact of paliperidone palmitate versus oral atypical antipsychotics in a Medicare population. J Comp Eff Res. 2018;7:723-35.

30. Clouth J, Liepa AM, Moeser G, et al. Hepatocellular carcinoma after prior sorafenib treatment: incidence, healthcare utilisation and costs from German statutory health insurance claims data. Health Econ Rev. 2018;8:18.

31. Mahlich J, Alba A, Hadad LE, et al. Drug survival of biological therapies for psoriasis treatment in Germany and associated costs: a retrospective claims database analysis. Adv Ther. 2019;36:1684-99.

32. Ziegelbauer K, Kostev K, Hubinger M, et al. The impact of nonpersistence on the direct and indirect costs in patients treated with subcutaneous tumour necrosis factor-alpha inhibitors in Germany. Rheumatology (Oxford). 2018;57:1276-81.

33. Gesetzliche Krankenversicherung - Kennzahlen und Faustformeln: Bundesministerium für Gesundheit. 2019. https://www.bunde sgesundheitsministerium.de/fileadmin/Dateien/5_Publikatio nen/Gesundheit/Broschueren/KF2019Bund_Maerz_2019.pdf. Accessed 17 Apr 2020

34. Bevölkerung auf Grundlage des Zensus 2011 stand: 30.09.2018: Destatis Statistisches Bundesamt. 2019. https://www.destatis.de/ DE/Themen/Gesellschaft-Umwelt/Bevoelkerung/Bevoelkerungsst and/Tabellen/liste-zensus-geschlecht-staatsangehoerigkeit.html. Accessed 17 Apr 2020

35. Haw C, Stubbs J. Off-label use of antipsychotics: are we mad? Expert Opin Drug Saf. 2007;6:533-45.

36. Cheung S, Hamuro Y, Mahlich J, et al. Drug utilization of Japanese patients diagnosed with schizophrenia: an administrative database analysis. Clin Drug Investig. 2017;37:559-69.

37. Mahlich J, Sruamsiri R. Persistence with biologic agents for the treatment of rheumatoid arthritis in Japan. Patient Prefer Adherence. 2016;10:1509-19.

38. Charlson ME, Pompei P, Ales KL, et al. A new method of classifying prognostic comorbidity in longitudinal studies: development and validation. J Chronic Dis. 1987;40:373-83.
39. Nau DP. Proportion of Days Covered (PDC) as a Preferred Method of Measuring Medication Adherence Springfield, VA: Pharmacy Quality Alliance. 2012. http://ep.yimg.com/ty/cdn/epill/pdcmp r.pdf. Accessed 17 Apr 2020

40. Campagna EJ, Muser E, Parks J, et al. Methodological considerations in estimating adherence and persistence for a long-acting injectable medication. J Manag Care Spec Pharm. 2014;20:756-66.

41. ATC/DDD German Institute of Medical Documentation and Information. 2020. https://www.dimdi.de/dynamic/de/arzneimitt el/downloads/?dir=atcddd. Accessed 9 Apr 2020

42. Sruamsiri R, Kameda H, Mahlich J. Persistence with biological disease-modifying antirheumatic drugs and its associated resource utilization and costs. Drugs Real World Outcomes. 2018;5:169-79.

43. Pilon D, Joshi K, Tandon N, et al. Treatment patterns in Medicaid patients with schizophrenia initiated on a first- or second-generation long-acting injectable versus oral antipsychotic. Patient Prefer Adherence. 2017;11:619-29.

44. Verdoux H, Pambrun E, Tournier M, et al. Risk of discontinuation of antipsychotic long-acting injections vs. oral antipsychotics in real-life prescribing practice: a community-based study. Acta Psychiatr Scand. 2017;135:429-38.

45. DiMatteo MR. Variations in patients' adherence to medical recommendations: a quantitative review of 50 years of research. Med Care. 2004;42:200-9.

46. Ljungdalh PM. Non-adherence to pharmacological treatment in schizophrenia and schizophrenia spectrum disorders-an updated systematic literature review. Eur J Psychiat. 2017;31:172-86.

47. Semahegn A, Torpey K, Manu A, et al. Psychotropic medication non-adherence and its associated factors among patients with major psychiatric disorders: a systematic review and meta-analysis. Syst Rev. 2020;9:17.

48. Kuwabara H, Saito Y, Mahlich J. Adherence and rehospitalizations in patients with schizophrenia: evidence from Japanese claims data. Neuropsychiatr Dis Treat. 2015;11:935-40.

49. Mahlich J, Olbrich K, Wilk A, et al. Hospitalization rates and therapy costs of German schizophrenia patients who are initiated on long-acting injectable medication: a mirror-image study. Clin Drug Investig. 2020;40:355-75.

50. Sruamsiri R, Mori Y, Mahlich J. Productivity loss of caregivers of schizophrenia patients: a cross-sectional survey in Japan. J Ment Health. 2018;27:583-7.

51. Mahlich J, Nishi M, Saito Y. Modeling the budget impact of longacting injectable paliperidone palmitate in the treatment of schizophrenia in Japan. Clinicoecon Outcomes Res. 2015;7:267-72.

52. Altamura AC, Aguglia E, Bassi M, et al. Rethinking the role of long-acting atypical antipsychotics in the community setting. Int Clin Psychopharmacol. 2012;27:336-49.

53. Correll CU, Citrome L, Haddad PM, et al. The use of long-acting injectable antipsychotics in schizophrenia: evaluating the evidence. J Clin Psychiatry. 2016;77:1-24. 


\section{Affiliations}

\section{Jörg Mahlich ${ }^{1,2}$ (I) Kerstin Olbrich ${ }^{1} \cdot$ Adrian Wilk $^{3} \cdot$ Antonie Wimmer $^{4} \cdot$ Claus Wolff-Menzler $^{5}$}

1 Health Economics and Outcomes Research, Janssen, Pharmaceutical Companies of Johnson \& Johnson, Neuss, Germany

2 Düsseldorf Institute of Competition Economics (DICE), University of Düsseldorf, Düsseldorf, Germany

3 Team Gesundheit, Gesellschaft für Gesundheitsmanagement $\mathrm{mbH}$, Essen, Germany
4 Medical Affairs, Janssen, Pharmaceutical Companies of Johnson \& Johnson, Neuss, Germany

5 Department of Psychiatry and Psychotherapy, University Medical Center Göttingen, Georg-August-University, Göttingen, Germany 